\title{
Statistical Mechanics of Nonlinear Wave Equations (4): Cubic Schrödinger
}

\section{H.P. McKean}

Courant Institute, New York University, 25I Mercer Street, New York, NY 10012, USA

Received: 10 July 1995

Commun. Math. Phys. 168, 479-492 (1995)

K. Vaninsky has kindly informed me that the application of the KolmogorovCentsov criterion to the tightness of the mean-values $M_{d}$ is not right. The criterion states that if $\mathbf{x}: t \in[0,1] \rightarrow x(t) \in \mathrm{R}$ is a random process with $E|\Delta x|^{\alpha} \leqq(\Delta t)^{\beta}$ for some $\beta>1$, then $|\Delta x| \leqq c(\mathbf{x})|\Delta t|^{\gamma}$ with probability 1 for any $\gamma>(\beta-1) / \alpha$. The same is true for a 2 -dimensional time $t \in[0,1]^{2}$, provided $\beta>2$ and $\gamma<(\beta-2) / \alpha$. The criterion was started for $d=1$ but applied to $d=2$; also the conclusion (midp. 485) has its exponents $1 / 4$ and $1 / 12$ inexplicably reversed. The correction is to improve the estimates pp. 483-485 and 489-490. Happily, this is easy. The Gaussian character of the reference measure $M_{0}$ leads, in the style of the original text, to the improved bounds:

$$
\begin{gathered}
M_{d}\left|e^{t X_{d}} Z(x+h)-e^{t X_{d}} Z(x)\right|^{2 n} \leqq c_{1}(n) h^{n}, \\
M_{d}\left|\left(e^{h X_{d}}-1\right) e^{t X_{d}} Z(x)\right|^{2 n} \leqq c_{2}(n) h^{n / 2}
\end{gathered}
$$

for any whole number $n$. The correct application of Kolmogorov-Čentsov requires $n=5$ or more. The use of $n \uparrow \infty$ and a refinement of the criterion produces the correct (and sharp) version of the display mid-p. 485: $|\Delta Z| \leqq c(Z)\left[(\Delta t)^{1 / 4-}+\right.$ $\left.(\Delta x)^{1 / 2-}\right]$. 